\title{
Optimisation de mélange amortissant par essais d'impact en laboratoire
}

PH. REIFFSTECK J.-L. TACITA

S. FANELLI

Université Paris-Est IFSTTAR

Gité Descartes 14-20, bd Newton Champs-sur-Marne 77447 Marne-la-Vallée Cedex 2
Il est envisagé d'employer des mélanges cailloux déchiquetats de pneumatiques usagés pour remplir les merlons pare-blocs utilisés en montagne comme protection contre les éboulements rocheux. Afin d'étudier la transmission de l'impact de ces blocs par une cellule remplie de mélange amortissant aux autres cellules, un programme d'essai complet a été réalisé en laboratoire. Cet article présente les résultats d'essais de compressibilité et de cisaillement effectués sur différents mélanges de sable avec des copeaux de pneus et une série d'essais d'impact en modèle réduit. Pour cela, un montage innovant a été réalisé et a permis d'évaluer l'effet d'amortissement avec des capteurs plats de pression. L'analyse des courbes tracées permet de déterminer un mélange aboutissant à un optimum d'amortissement.

Finalement, cet article propose des recommandations sur les fuseaux granulométriques d'acceptation spécifiques à ces matériaux hétérogènes amortisseurs et des données sur le comportement mécanique correspondant.

Mots-clés : essai d'impact, essai modèle réduit, étude expérimentale, remblai, matériau recyclé, protection.

\section{Optimization of damping mixed soil by impact tests in the laboratory}

It is proposed to use mixtures of used tires shreds and coarse gravels to fill the cells of rockfall protection structures used in mountain zones to protect homes against the blocks propagating from the cliffs. To study the transmission of these external impacts on a cell filled with this damping material, a complete test program was conducted in the laboratory.

This paper presents results of compression and shear tests performed on different mixtures of sand with tire chips and a series of impact tests on a reduced scale model. For this purpose an innovative assembly was developed allowing the damping effect to be evaluated with flat tactile pressure sensors. The analysis of the curves allows determining a mixture resulting in an optimum damping.

Finally this article proposes size acceptance criteria for the heterogeneous materials and data on the corresponding mechanical behaviour.

Key words: impact test, small scale test, experimental study, embankments, recycled material, protection. 


\section{Introduction}

Le dimensionnement des remblais renforcés composés de matériaux hétérogènes (sol de réemploi, déchets valorisés, matériaux dissipatifs) suscite des difficultés spécifiques mal résolues. Le projet REMPARe financé par l'ANR a tenté de répondre à cette problématique par l'utilisation de structure vraie grandeur et semi-grandeur qui sont essentiels à la compréhension des mécanismes en jeu et la validation des méthodes d'analyse développées (Haza-Rozier et al., 2010 ; REMPARe, 2010). Ces essais ne permettent pas toutefois par le faible nombre de massifs testés de faire varier les caractéristiques des matériaux amortisseurs constitutifs des massifs. En effet, le coût important de réalisation limite la reproduction Le programme de recherche présenté dans cet article a pour objectif une meilleure connaissance des caractéristiques mécaniques de ces matériaux hétérogènes en vue d'améliorer les modèles de calcul des ouvrages concernés. Ces recherches doivent permettre d'élaborer, à partir des outils disponibles ou à développer, des recommandations pour les reconnaissances (connaissance de la composition du sol et sa distribution) et pour les essais (en place et au laboratoire).

Afin de caractériser mécaniquement les mélanges, des essais de compressibilité et de cisaillement de laboratoire ont été réalisés sur des éprouvettes reconstituées avec des mélanges de sable avec des copeaux de pneus. Cette campagne d'essais paramétriques est une étape indispensable pour valider les hypothèses faites dans les simulations avec des modèles de mélanges et d'éléments distincts sur l'influence des effets d'échelle, de structure granulométrique et de nature des agrégats. Elle a été définie de manière à améliorer l'état des connaissances de ces matériaux déjà étudiés en laboratoire à la boîte de cisaillement direct, au triaxial, à la colonne résonnante ou lors d'essais en cuve (Edil et Bosscher, 1994; Masad et al., 1996 ; Feng et Sutter, 2000 ; Luong et al., 2003 ; Chikatamarla et al., 2005; Gotteland et al., 2005 ; Bałachowski et Gotteland, 2007 ; Mavroulidou et al., 2009). A la suite de cela, des essais d'impact sur une cellule remplie des différents mélanges ont permis d'évaluer l'effet amortissant en fonction de la proportion des composants. Finalement une synthèse de ces différents essais permet de proposer des dosages adaptés à cette application en merlon pare-blocs.

\section{2}

\section{Caractéristiques mécaniques}

\section{1 \\ Granulométrie des matériaux et des mélanges}

Le sable choisi pour cette expérience est le sable de Fontainebleau qui est un sable de référence aux caractéristiques connues (Mestat, 2000). A partir de la courbe granulométrique (Fig. 1) obtenue par la méthode par tamisage à sec après lavage, on trouve que ce sable provenant d'une carrière située à Fontainebleau est mono-granulaire, avec $\mathrm{d}_{\max }=0,4 \mathrm{~mm}$ et $\mathrm{d}_{50}=0,2 \mathrm{~mm}$. Le coefficient d'uniformité $\mathrm{C}_{\mathrm{U}}$ est égal à 1,53 et celui de courbure $\mathrm{C}_{\mathrm{C}}$ à 0,93 . La mesure au pyc- nomètre à hélium de marque Quantachrome Instruments de la masse volumique des grains du sable de Fontainebleau est 2,65 $\mathrm{g} / \mathrm{ml}$.

Le matériau simulant les déchiquetats de pneumatiques est obtenu à partir de déchiquetats réels utilisés dans le cadre du projet REMPARe réduits à la taille de copeaux de formes cubiques de côté 2 à $5 \mathrm{~mm}$. Pour vérifier l'homogénéité géométrique des copeaux, ils sont passés dans des tamis de maille de 2 et $5 \mathrm{~mm}$.

La mesure au pycnomètre à hélium de la masse volumique d'un morceau de pneu donne $1,13 \mathrm{~g} / \mathrm{ml}$ ce qui est très légèrement différent de la masse volumique des copeaux de pneu égale à $1,164 \mathrm{~g} / \mathrm{ml}$.

Les mélanges qui feront l'objet des expérimentations décrites dans cet article sont élaborés en faisant varier le pourcentage massique de sable et de pneu : $100 \%$ sable, $75 \%$ sable $25 \%$ pneu, $50 \%$ de sable et de pneu, $25 \%$ sable $75 \%$ pneu, $100 \%$ pneu.

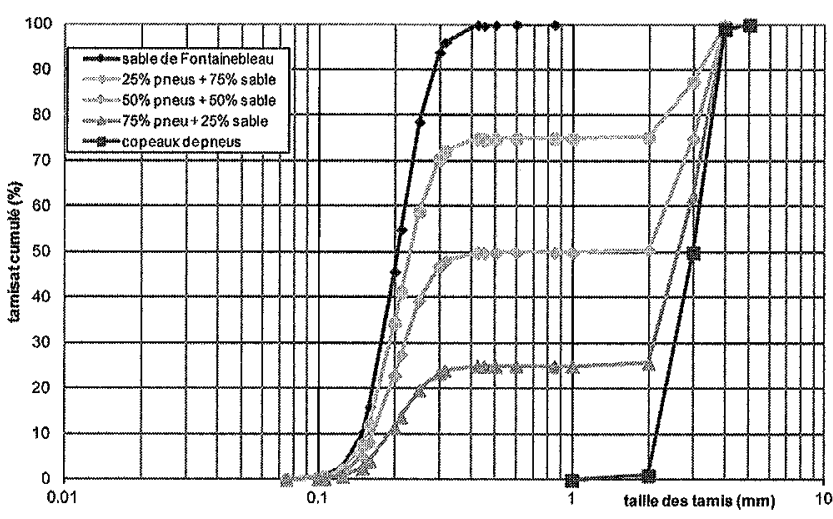

16. 1. Courbes granulométriques des mélanges sable de Fontainebleau, copeaux de pneumatiques.

Particle size distribution of Fontainebleau sandtire shreds mixture.

Les courbes granulométriques obtenues présentent de ce fait un palier. Le rapport entre les valeurs des diamètres moyens $\mathrm{d}_{50}$ des deux matériaux constitutifs est de 15 permettant au sable de prendre place lors du mélange dans les interstices, ou pores, entre les copeaux de pneumatiques. On estime en effet à partir de résultats expérimentaux sur des mélanges binaires de matériaux mono-granulaires que ce rapport doit être au minimum de 7 pour que les particules de plus petite taille s'insèrent dans les pores de la fraction grossière (Lade et al., 1998 ; Reiffsteck et al., 2007b).

La figure 2a présente l'évolution de la masse volumique moyenne des grains mesurée au pycnomètre à hélium en fonction de la proportion des mélanges. Elle évolue non linéairement mais sans variation brusque notable. La masse volumique des mélanges à l'état lâche dépend de l'indice des vides et donc de l'arrangement granulaire. Les courbes de la figure $2 \mathrm{~b}$ présentent une variation de pente au-delà de $50 \%$ de pneumatique. 

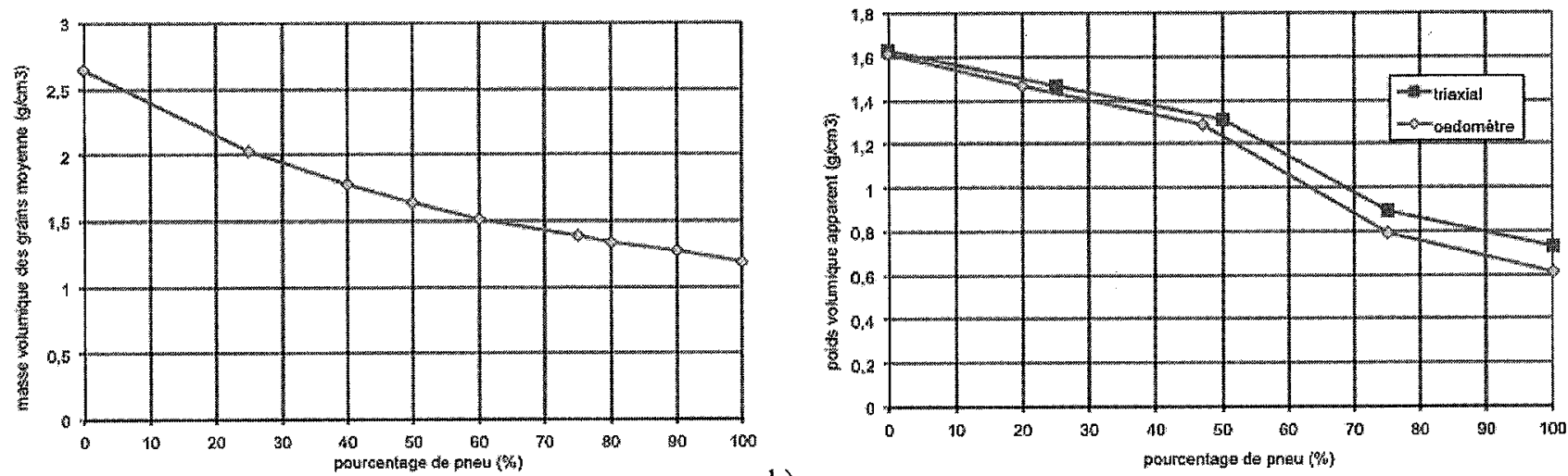

a)

b)

a) Masses volumiques des grains moyennes et b) poids volumique apparent des mélanges sable de Fontainebleau, copeaux de pneumatiques.

a) Mean particle density and b) bulk density of Fontainebleau sand - tire shreds mixture.

\section{2.}

\section{Compressibilité des mélanges}

Des essais œdométriques réalisés sur cinq mélanges secs à deux composants avec le pourcentage de pneu variant de $25,50,75$ et $100 \%$ ont été effectués pour observer l'évolution de la compressibilité. Ils ont été réalisés conformément à la norme NF P94-090-1 sur des éprouvettes de diamètre $60 \mathrm{~mm}$. La taille des plus gros copeaux reste inférieure au dixième du diamètre de la bague. Toutefois, la hauteur ne respecte pas le critère des six diamètres requis par cette norme mais comme il ne s'agit pas d'inclusions rigides comme sous-entendu par la norme, il n'y a pas eu de modification du matériel d'essais (Reiffsteck et al., 2007a).
Ces essais ont été répétés deux fois (quatre fois pour le sable) pour s'assurer de la répétabilité de la procédure de mise en place dans la bague œdométrique.

La figure 3 met en évidence deux familles de courbes avec une transition assez rapide pour un pourcentage compris entre 50 et $75 \%$. De ce fait, il semble possible à partir des résultats des essais œdométriques de trouver la proportion correspondant à un meilleur arrangement.

On observe que les cycles de déchargement rechargement présentent la même concavité que la courbe de premier chargement du fait de l'absence d'effet différé.

Un retour élastique important est également observé proche de celui obtenu sur des sols gonflants. Cet aspect est bien sûr maximal pour le mélange $100 \%$



Relations contrainte vertical effective et indice des vides des mélanges.

Effective vertical stress versus void ratio of the mixtures. 
pneu qui se comporte comme l'élastomère qui le compose, la faible irréversibilité provenant sans doute du réarrangement des copeaux.

A noter que pour les plus fortes contraintes, l'indice des vides devient très faible et les courbes de compression présentent une inversion de courbure typique des structures alvéolaires comprimées perpendiculairement à leur plan d'isotropie.

Le tableau I donne les valeurs des pentes des courbes (indice de compression et de gonflement) ainsi que le module œdométrique mesuré sous une charge de $100 \mathrm{kPa}$.

La figure 4 montre que la variation des indices de compression et de recompression présente une rupture de pente pour une valeur de dosage correspondant environ à $60 \%$ de sable et $40 \%$ de copeaux de pneumatiques. L'étude menée par Mavroulidou et al. (2009) aboutit à des résultats très similaires.

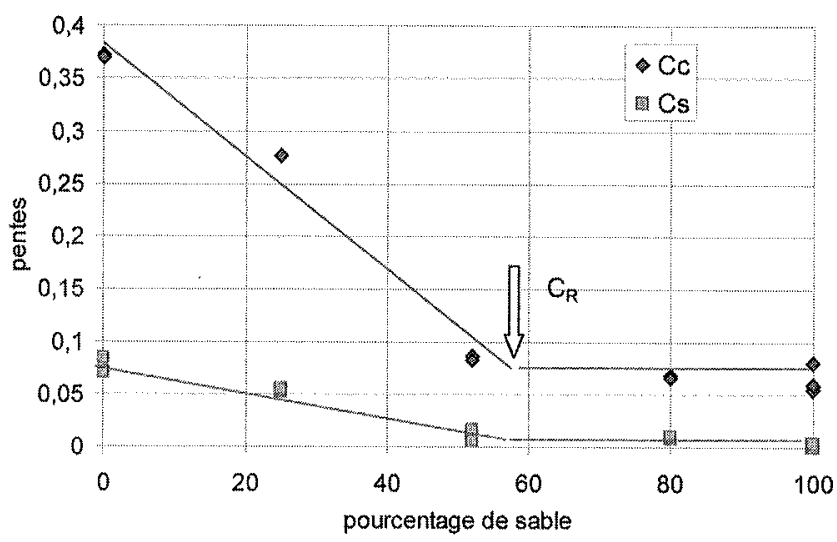

15- 16.4 Relations entre les pentes des essais de compressibilité et les proportions des mélanges.

Relation between slopes of compressibility tests and mixture proportions.

\section{Caractéristique de cisaillement des mélanges}

Afin d'étudier l'évolution des caractéristiques de cisaillement avec les proportions des composants, cinq mélanges avec le pourcentage de pneumatique variant de 25,50, 75 et $100 \%$ ont été réalisés et testé à l'état sec à l'appareil triaxial. Les essais sont des essais non consolidés non drainés (UU) réalisés selon la norme NF P94-074. Les éprouvettes ont été reconstituées au préalable dans un moule métallique sécable directement dans l'appareil triaxial avec une dimension de $100 \mathrm{~mm}$ de hauteur, de $50 \mathrm{~mm}$ de diamètre. Les mélanges ont été mis en place à la cuillère avec une hauteur de chute constante puis légèrement vibrés pour limiter les difficultés au démoulage réalisé avec une dépression.

Une mesure des indices des vides minimal et maximal selon la norme NF P94-059 a été réalisée sur les copeaux de pneumatique et le sable de Fontainebleau (tableau II). L'indice de densité de mise en œuvre vaut 0,85 pour le sable et 0,20 à 0,73 pour les copeaux pour l'essai triaxial et cedométrique respectivement (tableau III). Malgré l'application de procédures normalisées, le matériau copeaux pur n'a pu être mis en œuvre à un indice des vides suffisamment élevé au triaxial au risque d'obtenir une éprouvette très instable pour le montage (Fig. 5).

Avec un mélange à deux composants, on trouve que quand le pourcentage de particules fines, ici le sable, est de $0 \%$, l'indice des vides du mélange est

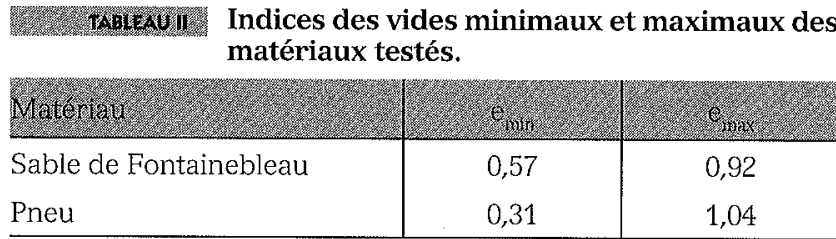

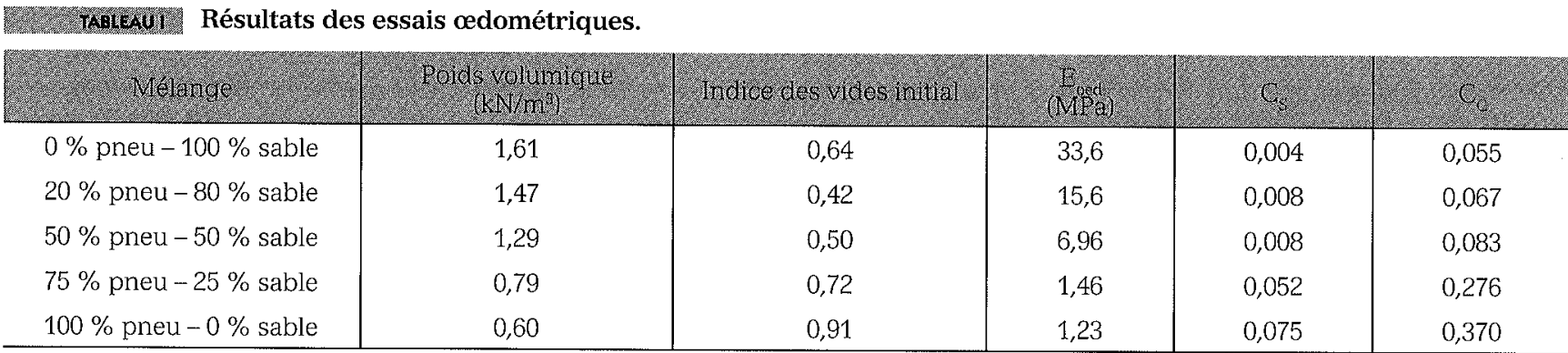

TAELAUIII Résultats des essais triaxiaux.

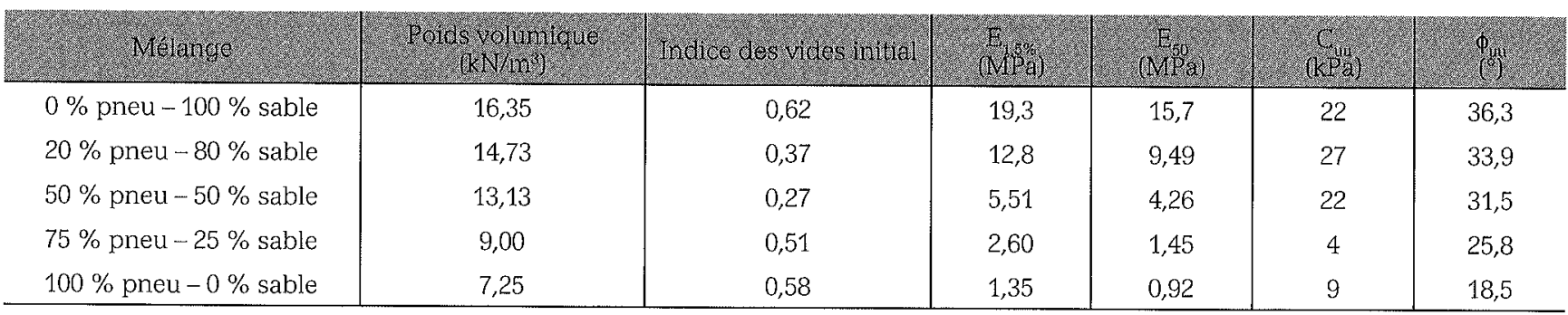


celui des grosses particules, les copeaux. Quand on augmente un peu le pourcentage des particules fines, celles-ci s'insèrent dans les vides entre les gros grains, l'indice des vides diminue, passe par un minimum puis augmente (Fig. 5). L'indice des vides minimum $\left(e_{\min }\right)$ est atteint quand les vides entre les gros grains sont remplis des particules fines (Lade et al., 1998 ; Reiffsteck et al., 2007b). Le pourcentage à ce seuil est noté Cr sur la figure 5.



Hers Évolution de l'indice des vides pour les différents mélanges.

Variation of void ratio for the various mixture.

Pour des pourcentages inférieurs à $30 \%$, une forte influence de l'indice des vides est observable. Il semble que pour un pourcentage de sable supérieur, le comportement est piloté par le squelette granulaire.

Finalement, le cisaillement non drainé a été réalisé à trois pressions effectives de confinement $\sigma_{3}^{\prime}: 50$, 100 et $150 \mathrm{kPa}$, respectivement à une vitesse de déformation de $0,6 \% / \mathrm{min}$, ce qui est la valeur basse de la vitesse préconisée pour réaliser l'essai UU. Les essais ont été poursuivis jusqu'à une déformation de $15 \%$ voire jusqu'à $25 \%$ pour obtenir un pic (Fig. 6).

On observe une évolution continue des courbes effort-déformation des éprouvettes constituées uniquement de sable vers celles constituées uniquement de copeaux de pneumatiques. Pour chaque pression de confinement, la courbe correspondant à un mélange à $50 \%-50 \%$ reste proche du groupe $100 \%$ de sable et $75 \%$ sable $-25 \%$ copeaux.
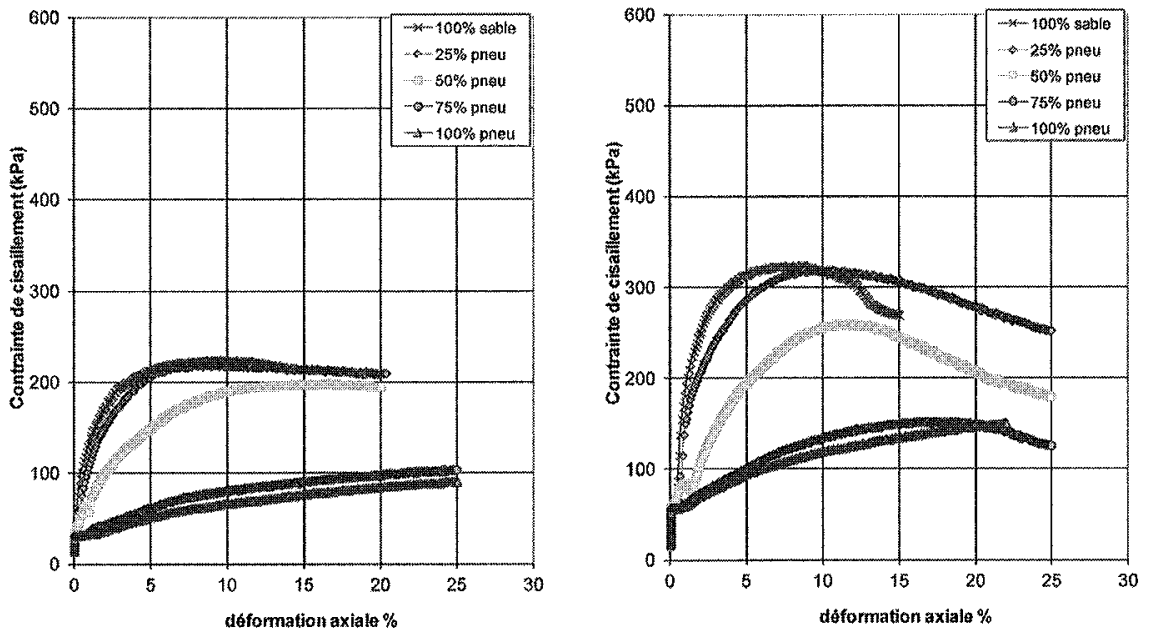

On peut en première approche conclure que le changement de comportement s'effectue à un pourcentage entre $50 \%$ et $25 \%$ de sable.

Les modules déduits des essais œdométriques et triaxiaux évoluent en fonction du pourcentage de sable (Fig. 7).



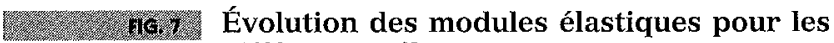
différents mélanges.

Evolution of elastic modulus for the various mixtures.

Le module d'Young obtenu au triaxial sur l'éprouvette composée uniquement de copeaux de caoutchouc confinée à $100 \mathrm{kPa}$ est de 1,15 MPa, valeur que l'on peut comparer au module d'Young du caoutchouc plein évalué à $10 \mathrm{MPa}$.

Les résultats obtenus sont en accord avec l'étude menée par Masad et al. (1996) avec des granulométries de copeaux et de sable très similaires à celles de l'étude présentée et celle faite Feng et Sutter (2000) en petites déformations. Nous avons également représenté les résultats de Bałachowski et Gotteland (2007) obtenus au triaxial sur des chips à forte granulométrie dans la configuration de dépôt aléatoire. Cette étude menée en amont du projet REMPARe permet d'avoir des informations avec des tailles de déchiquetats proches de celles utilisées dans les ouvrages réels.

Les valeurs proposées par Mavroulidou et al. (2009) sont très faibles, toutefois les conditions d'essai et la nature exactes des mélanges représentés sur les figures ne sont pas précisés.

Ce constat peut également être fait sur la figure 8 qui met en évidence l'évolution de l'enveloppe de rupture au pic avec les pourcentages de copeaux de pneumatiques dans l'éprouvette.

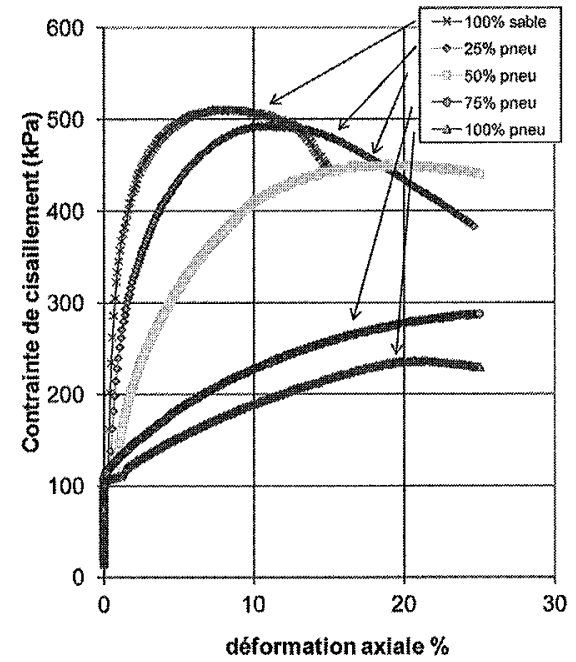

19. 6 Relations $q-\varepsilon 1$ des mélanges à 50, 100 et $150 \mathrm{kPa}$ de contrainte de confinement. Relation deviator versus vertical strain of mixture for 50,100 and $150 \mathrm{kPa}$ of confining stress. 




He. : Relations $s^{\prime}-t$ des différents mélanges. Failure envelopes for the different mixtures.

La figure 9 et le tableau III montrent que l'évolution de l'angle de frottement en fonction du pourcentage de copeaux de pneumatiques est continument décroissante. Cette observation a également été faite par Masad et al. (1996). Les essais triaxiaux drainés réalisés par Gotteland et al. (2005) et Zornberg et al. (2004) avec chips de caoutchouc de grande taille par rapport au triaxial donnent la même tendance. Ils ont également constaté une grande importance de l'orientation des particules dans l'éprouvette. Les mesures réalisées à la boîte de cisaillement par Edil et Bosscher (1994) ne semblent pas en accord avec ces résultats. On peut toutefois observer que la taille importante des copeaux de pneumatiques testés dans la boîte a pu perturber les mesures réalisées par ces auteurs (Reiffsteck et al., 2007a).

La cohésion apparente observée (à l'exception des valeurs données par Masad et al., 1996) semble maximale pour l'état qui a été observé comme le plus dense (Fig. 5).

La cohésion apparente du sable est probablement liée à la réalisation de l'essai en condition UU avec une vitesse de chargement élevée nuisant à l'apparition de la localisation des bandes de cisaillement au sein de l'éprouvette. Cependant, ce type d'essai est sans doute plus représentatif des conditions de chargement rapide que subiraient des ouvrages étudiés.

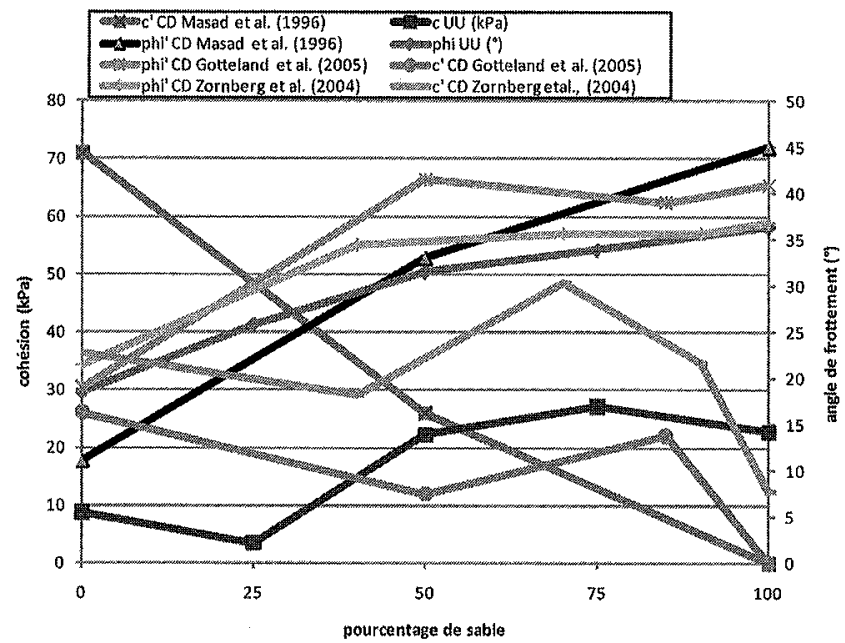

Valeurs des paramètres de rupture pour les différents mélanges.

Failure parameters obtained for different mixtures.

\section{Essais d'impact}

31

\section{Principe de l'essai}

L'essai d'impact, développé spécifiquement pour cette étude, consiste à solliciter une éprouvette de forme cubique de côté $10 \mathrm{~cm}$ par un impact localisé sur une face ; les autres faces étant confinées (Fig. 10). Le confinement apporté par la cellule (coffrage et chapeau sur l'éprouvette) a pour objectif de simuler les conditions aux limites d'une cellule environnée par d'autres cellules au sein d'un merlon pare-blocs.

L’impacteur est une demi-sphère métallique projetée contre la face de l'éprouvette au moyen d'un vérin pneumatique horizontal. L'ensemble est attaché à un socle pour un meilleur maintien.

Il est possible d'estimer l'énergie de l'impact à partir de la variation d'énergie cinétique subie par l'impacteur au moment de la frappe. La pression P s'exerçant sur le piston de surface $S$ crée une force $F$ qui provoque un déplacement de ce dernier. Au moment de l'impact, l'énergie cinétique de la tige est égal au travail de la force extérieure $F$ :

tel que :

$$
\begin{gathered}
\Delta \mathrm{E}=\sum \mathrm{W}\left(\mathrm{F}_{\mathrm{ext}}\right) \\
\frac{1}{2} m_{X} V^{2}=P_{X} S_{X} L
\end{gathered}
$$

avec :

- S la section supérieure du piston subissant la pression de l'air comprimé ;

- P la pression de l'air comprimé ;

- L la course du piston ;

- m la masse du piston.

D'où on peut tirer une expression simplifiée de la vitesse au moment du choc :

$$
V=\sqrt{\frac{2 \times(P \times S) \times L}{m}}=8,7 \mathrm{~m} / \mathrm{s}
$$

L'estimation de la vitesse d'impact peut être faite connaissant la pression de $700 \mathrm{kPa}(1 \mathrm{~Pa}=$ $0,10194 \mathrm{~kg} \cdot \mathrm{m}^{-1} \cdot \mathrm{s}^{-2}$ ) appliquée sur une surface intérieure du vérin de $5,01 \mathrm{~cm}^{2}$, de la masse de la tige du vérin de course de $160 \mathrm{~mm}$ équipé d'un capteur et de la sphère évaluée à $0,150 \mathrm{~kg}$. Ce qui donnerait une énergie d'impact de $E=5,7 \mathrm{~J}\left(\mathrm{~kg} \cdot \mathrm{m}^{2} / \mathrm{s}^{2}\right)$, valeur bien inférieure au $10 \mathrm{~kJ}$ mis en œuvre dans les expérimentations semigrandeur (Haza-Rozier et al., 2010). Toutefois ces expérimentations n'ont pas pour but de respecter les lois de la similitude mais de fournir un cadre de référence qualitatif.

Le système de mesure comportant sept capteurs permet de suivre l'impact (Fig. 10) :

- un capteur de force placé sur la tige du vérin horizontal mesure la force d'impact ;

- un capteur de pression placé sur le boîtier permet de mesurer la stabilité de la dépression appliquée à l'éprouvette ;

- cinq capteurs plats de pression ou capteurs de contact (Fig. 11). Ils sont placés sur les trois faces verticales du boîtier pour mesurer la réaction de l'éprouvette vis-à-vis de l'impact. 


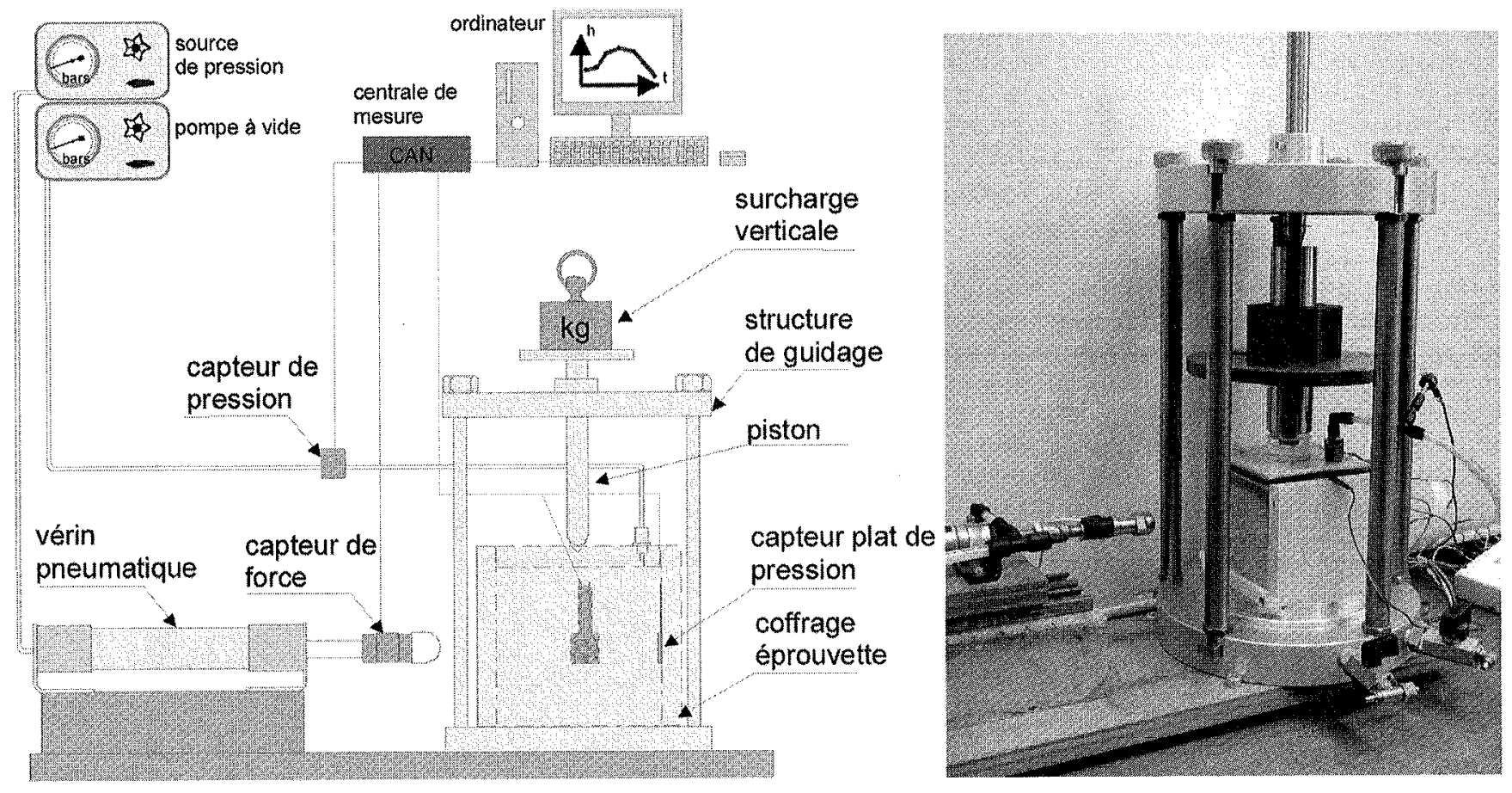

16. 10. Schéma du dispositif d'impact sur éprouvettes. Apparatus used to impact cubic samples.

L'acquisition des mesures est réalisée par un système d'acquisition constitué :

- d'un boîtier d'acquisition National Instruments (NI USB-6211) suffisamment rapide pour les mesures envisagées ;

- d'un ordinateur avec un programme spécifiquement écrit pour l'application sous l'environnement Labwindows de National Instruments.

Les valeurs sont acquises par le programme toutes les centièmes de seconde pendant la durée de l'essai et sont enregistrées au format texte.

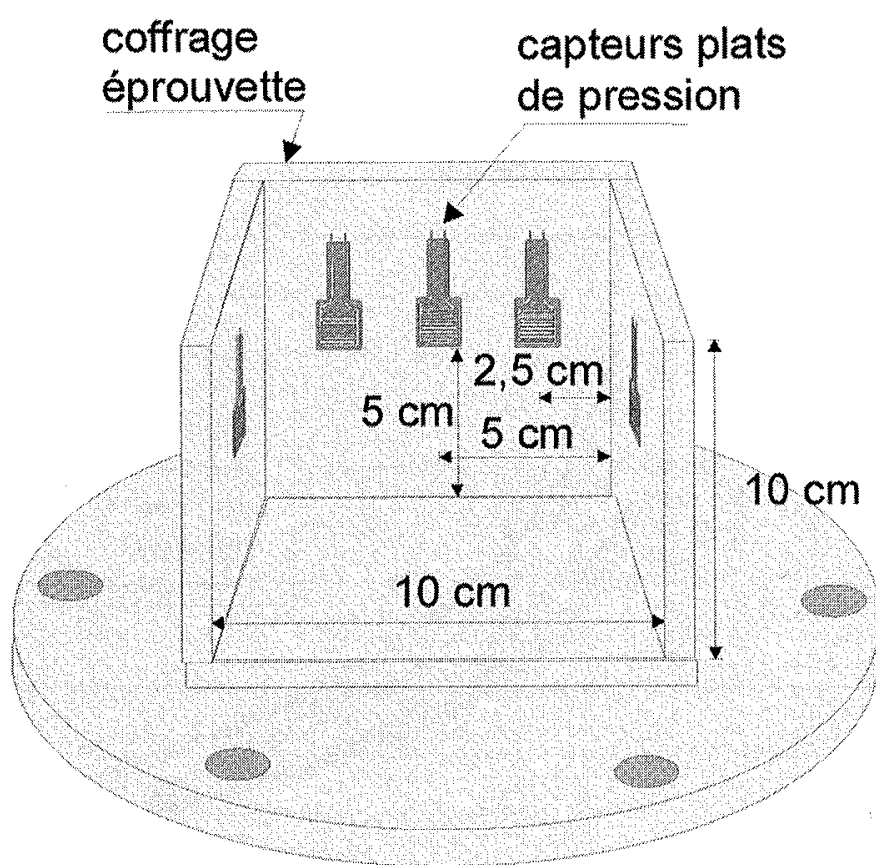

3.2.

\section{Description des résistances de détection de force}

Les capteurs plats de pression, ou résistance de détection de force pour Force Sensitive Resistors (FSR) en anglais, sont des capteurs tactiles initialement mis au point pour les claviers d'instruments de musique afin de moduler le son en fonction de la force de frappe sur les touches. Leur sensibilité à la force est optimisée pour la commande tactile d'appareils électroniques et

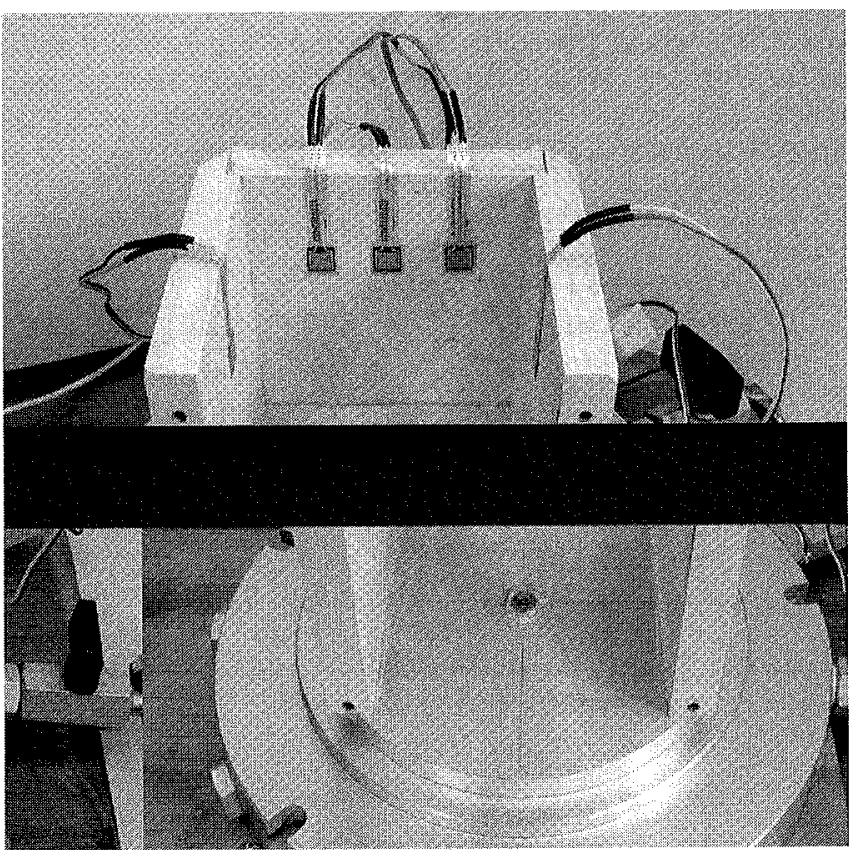

176. 11. Position des cinq capteurs plats de pression. Localisation of the five flat pressure transducers. 
mécaniques dans les plages de pressions à l'échelle humaine (de 0,1 à 1 bar). Ce type de capteur est plus simple d'utilisation que les capteurs Tekscan utilisés par Paikowskiet Hadjuk (1997) et Chikatamarla et al. (2005).

Il s'agit de composants dont la résistance décroît d'autant plus que la force appliquée perpendiculairement à sa surface augmente. Ce type de capteur présente une hystérésis plus faible que le caoutchouc conducteur et est moins sensible aux vibrations que les capteurs à couche piézoélectrique. Un capteur élémentaire se compose de deux feuilles de polymère laminées ensemble. L'une des feuilles est recouverte d'un réseau d'électrodes à plages intercalées, l'autre d'un matériau semi-conducteur (Fig. 12). Lorsqu'une force est appliquée sur le composant, le matériau semi-conducteur shunte plus ou moins les électrodes à plages intercalées. Le temps de montée mécanique est normalement de 1 à 2 millisecondes environ. Ces capteurs présentent une dégradation minime de performance $(5 \%)$ après dix millions de cycles.

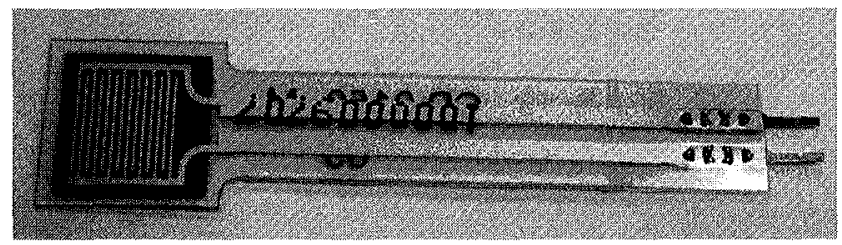

16. 11. Capteur plat de pression.

Force sensitive resistors.

Les capteurs choisis pour les essais ont une surface de mesure de $1 \mathrm{~cm}$ de côté. Ces composants peuvent avoir jusqu'à $55 \mathrm{~cm}$ de large sur $75 \mathrm{~cm}$ de long et ont une épaisseur variant entre 0,20 et $0,75 \mathrm{~mm}$.

La figure $13 a$ donne un exemple de réaction des capteurs lorsqu'ils sont pressés manuellement.

Les capteurs ont été étalonnés sur des chemins croissant et décroissant de chargement. La relation observée en la pression appliquée et la mesure est logarithmique (Fig. 13b). Toutes les relations ont été calées avec un coefficient de détermination compris entre 0,88 et 0,98 .

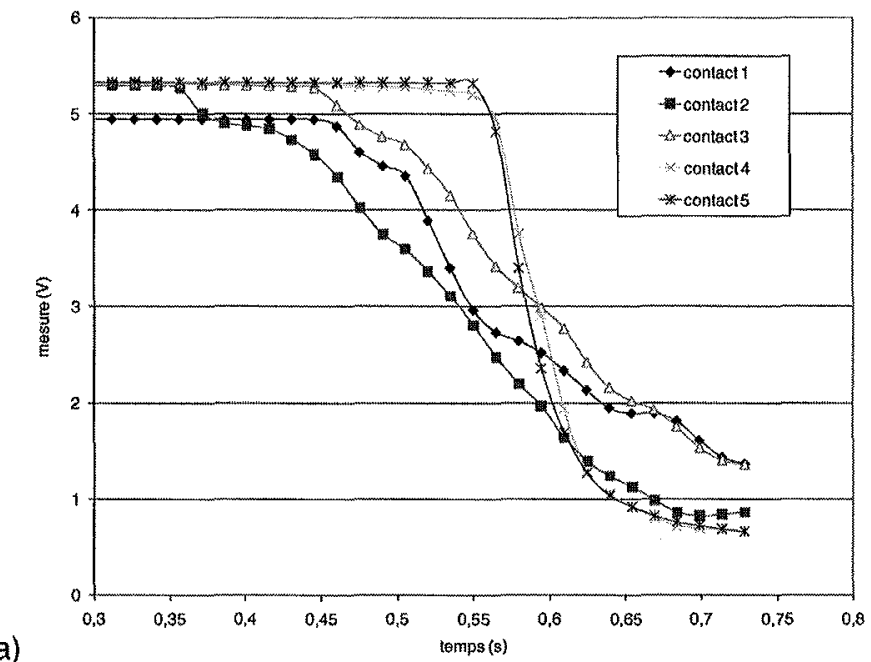

La position des cinq capteurs plats sur les parois est donnée sur la figure 14. Trois sont placés au milieu des faces verticales et les deux derniers capteurs en complément sur la face verticale du fond entre le milieu de la face et l'arête.

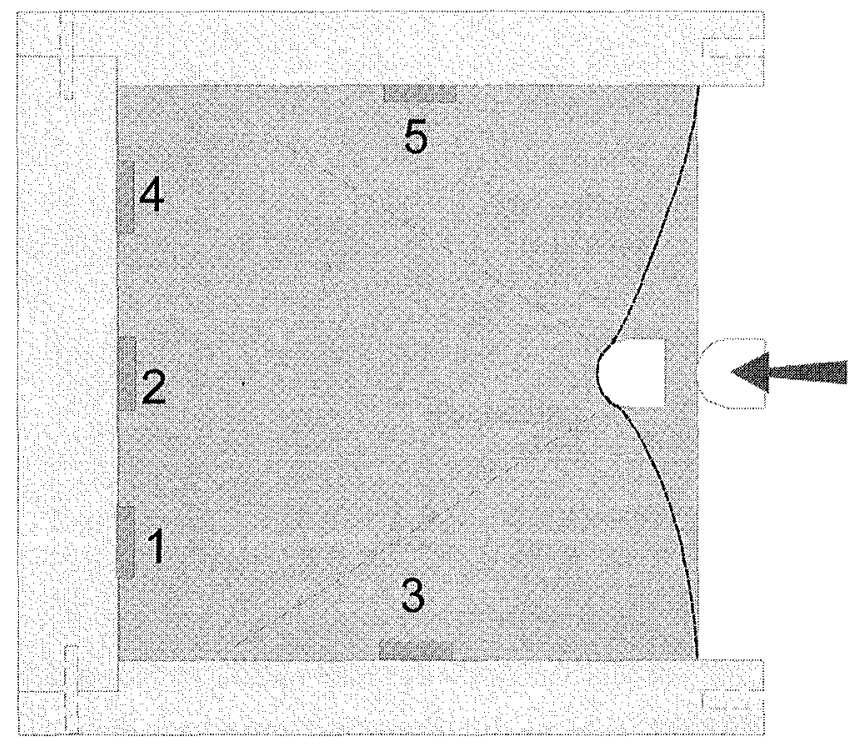

月6. 14 Positionnement des capteurs plats de pression.

Location of flat pressure transducers.

\section{Réalisation des essais}

Les mélanges sont placés dans une membrane en latex habituellement utilisée pour les essais de type triaxiaux placée à l'intérieur des trois parois du dispositif.

Lors de la reconstitution des éprouvettes, une plaque amovible vient fermer les trois plaques pour créer un moule cylindrique à base carrée. Les mélanges sont déposés manuellement avec une hauteur de chute nulle puis légèrement vibrés par des chocs sur les parois du dispositif. Le chapeau est placé sur les mélanges et la membrane est maintenue sur celui-ci par un joint torique.

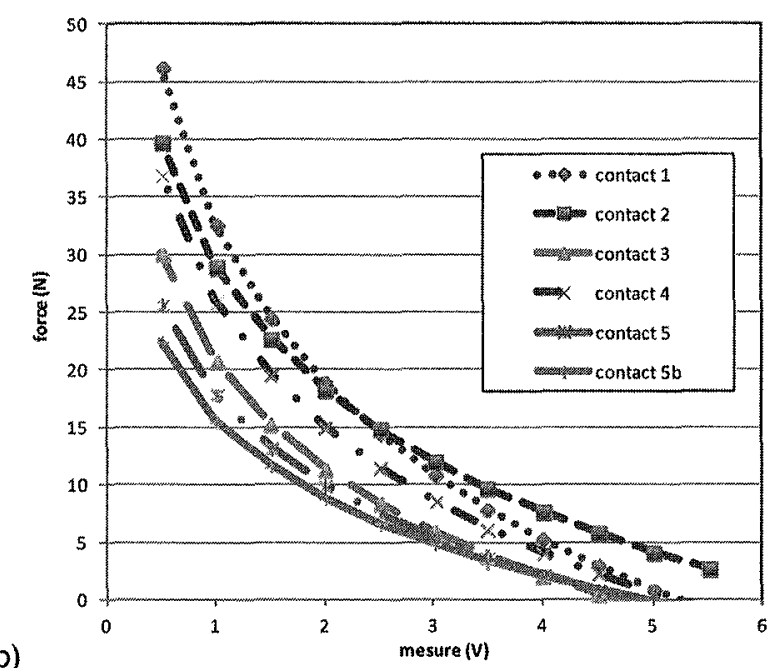

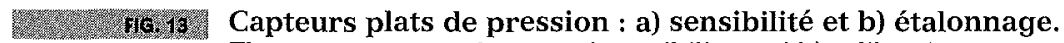
Flat pressure transducers : a) sensibility and b) calibration. 
Les essais étant réalisés avec le matériau sec, un léger vide permet de simuler un confinement produit par les structures de grillage métallique.

Après avoir retiré la face amovible, le piston vertical est mis en contact pour appliquer un chargement équivalent à une cellule supplémentaire.

\section{4}

\section{Déclenchement de l'impact}

Après le lancement du logiciel d'acquisition, l'impact est déclenché par mise en contact de la chambre du vérin et le réseau d'air comprimé par action manuelle sur une vanne. La phase d'impact est très brève de l'ordre du dixième de seconde.

\section{Programme expérimental}

Le plan d'expérience a consisté principalement à examiner l'influence des pourcentages respectifs des fractions granulométriques. Lors des premiers essais, il a été constaté une certaine dispersion des résultats. De ce fait, les essais d'impact ont été répétés de quatre à huit fois.

\section{1}

\section{Résultats et analyses}

Les figures 15 à 17 regroupent trois exemples des résultats d'essais. Sur ces graphiques, l'évolution des forces mesurées a été représentée en fonction du temps. Afin d'observer la réactivité de tous les capteurs, les valeurs des pressions mesurées sont normalisées par la valeur finale. Sur l'autre graphique (les figures notées b), les mesures ont été mises à l'échelle.

Les figures montrent que tous les capteurs réagissent mais pas avec la même amplitude et ceci en fonction de la nature du mélange sur lequel l'impact est réalisé. Il semble que des répartitions des déformations privilégiées apparaissent et que des voûtes peuvent se former, comme illustré sur la figure 14.

Lors des essais sur les éprouvettes avec $100 \%$ de sable le détecteur 2 (celui au milieu de la face du fond) n'a rien détecté du fait d'un dysfonctionnement. Son signal n'a donc pas été représenté.

Les signaux sont croissants puis restent stables sur un plateau contrairement aux essais pendulaires menés par Haza-Rozier et al. (2010) et Lambert et al. (2010) ou gravitaires réalisés ou décrits par Lambert (2007) et Gotteland et al. (2007) qui présentent tous une décroissance du signal liée à la dissipation de l'énergie cinétique par l'endommagement de l'ouvrage. Les impacts imposés par le vérin ont un caractère moins impulsif que les impacts pendulaires ou gravitaires. La plus faible énergie de l'impact et la taille des grains ne permettent pas un réarrangement et une rupture de ceux-ci.

Pour un pourcentage de sable inférieur à $60 \%$ ou de caoutchouc supérieur à $40 \%$ les signaux obtenus au niveau des capteurs plats deviennent plus importants (Figs. 15b et 17b). On constate que pour le dosage $100 \%$ de sable, il n'existe pas de différences notables entre les arrivées d'ondes de compression sur les différents capteurs. Dès l'ajout de copeaux de caoutchouc, une asymétrie est constatée. Certains capteurs observent l'impact avec un retard (contact 2 à $60 \%$ - figure $16 a$ - et 3 et 4 à $100 \%$ - figure $17 \mathrm{a}$ - de pneu par exemple).

Ces observations semblent en accord avec celles faites par Lambert et al. (2010) sur les merlons du Centre d'expérimentations routière de Rouen. La contrainte transmise dans l'ouvrage était supérieure avec le noyau en mélange sable-pneumatique qu'avec le sable pur et la transmission est retardée pour l'ouvrage contenant des déchiquetats de pneumatiques.

Lors des essais sur les éprouvettes de pneu (100\% de pneu), le vérin s'enfonce beaucoup plus facilement et la membrane a tendance à se déchirer. Ceci peut avoir faussé les résultats.

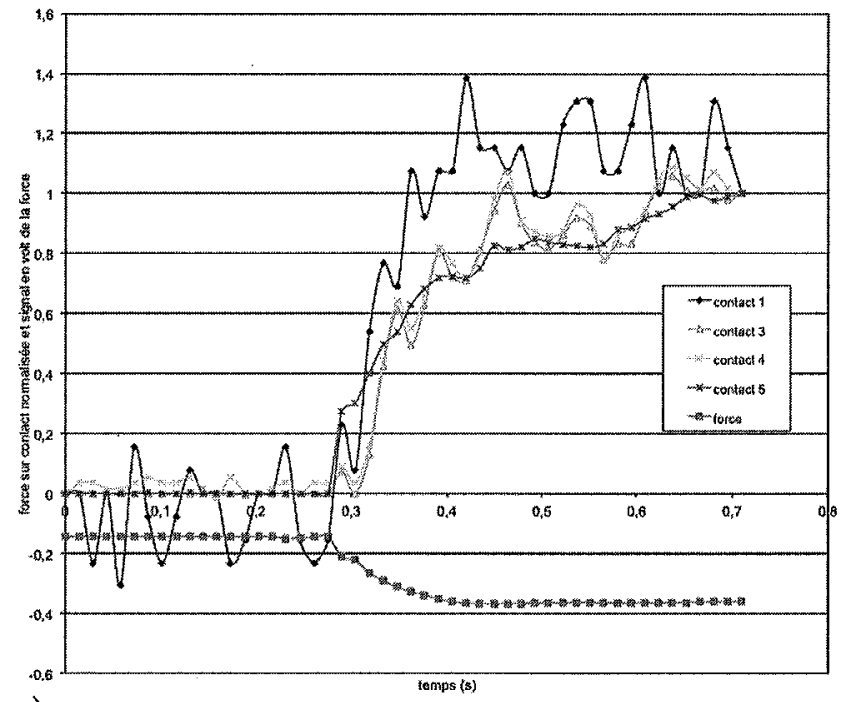

a)

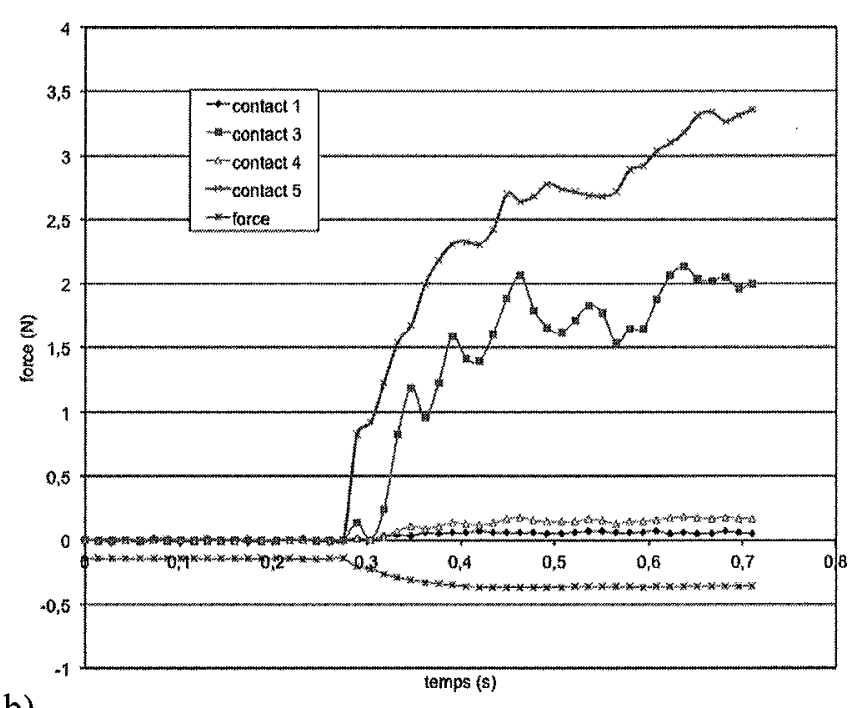

b)

Résultats d'essais d'impact pour un mélange de $100 \%$ sable - $0 \%$ pneu : a) signal brut, b) mesures mises à l'échelle.

Results of impact tests for a mixture of sand $100 \%-0 \%$ tire, a) raw signal, b) measures scaled. 

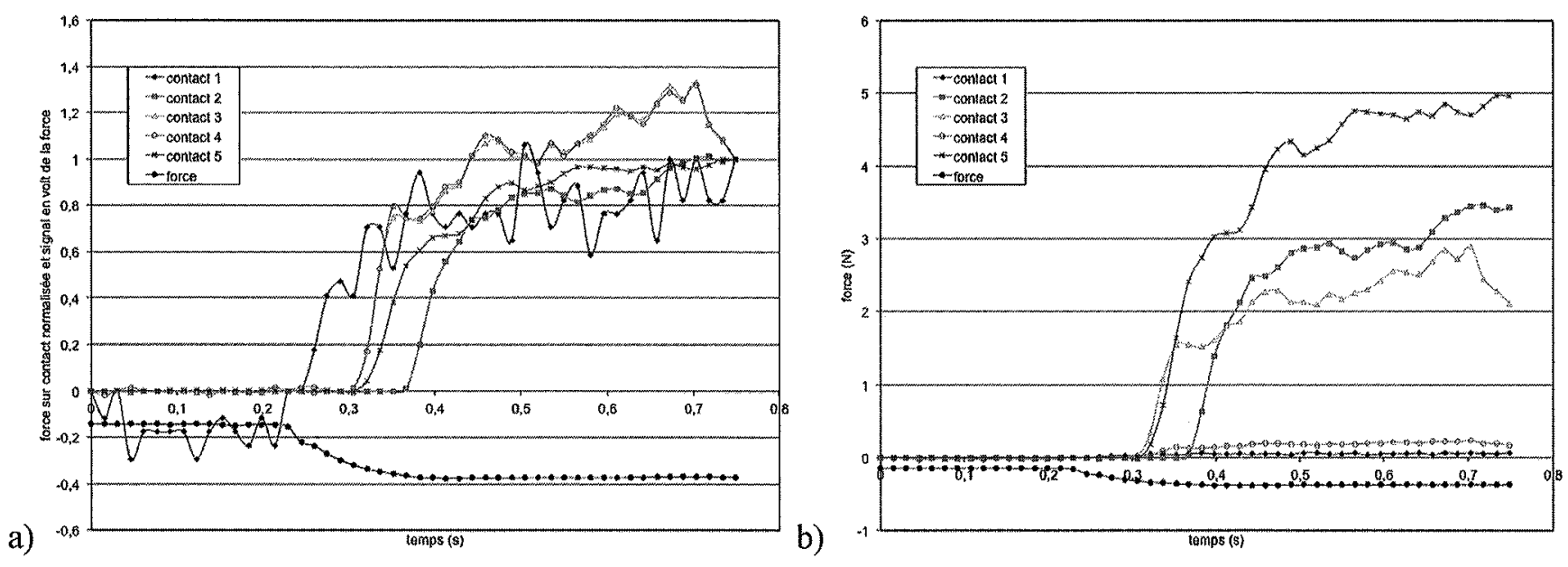

Résultats d'essais d'impact pour un mélange de $60 \%$ sable - $40 \%$ pneu, a) signal brut, b) mesures mises à l'échelle.

Results of impact tests for a mixture of sand $60 \%-40 \%$ tire, a) raw signal, b) measures scaled.
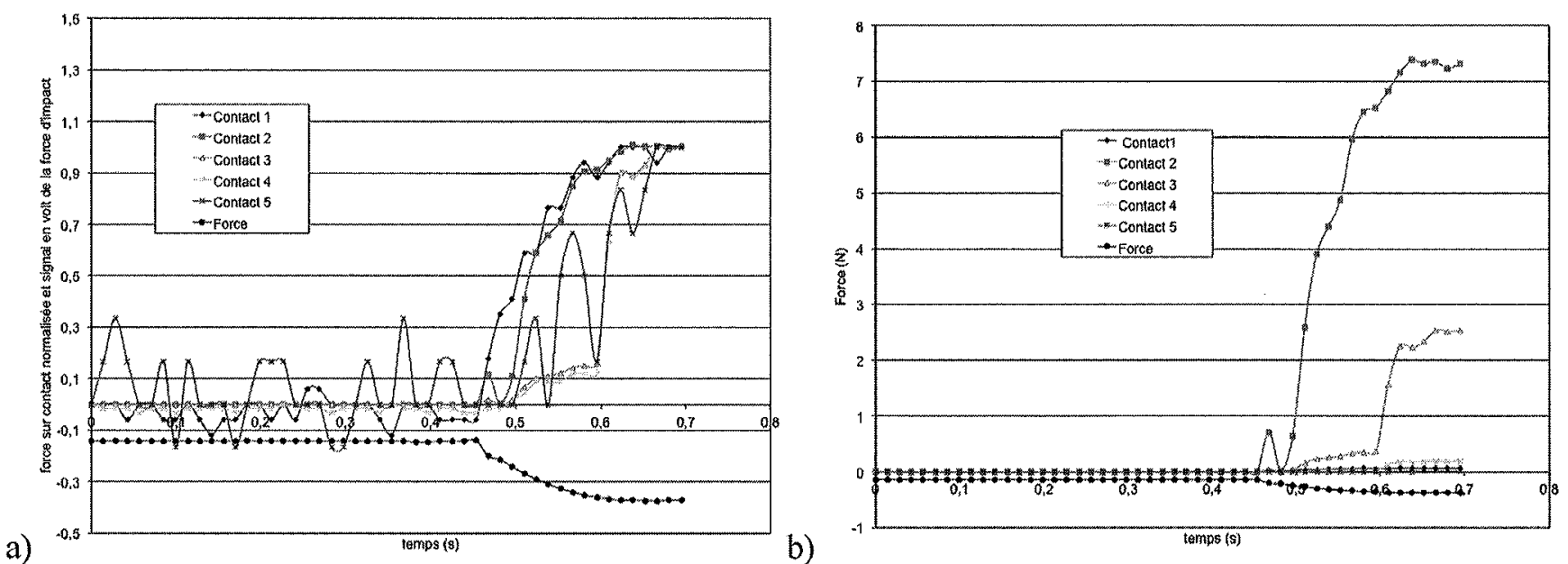

b)

Résultats d'essais d'impact pour un mélange de $0 \%$ sable - $100 \%$ pneu, a) signal brut, b) mesures mises à l'échelle.

Results of impact tests for a mixture of sand $0 \%-100 \%$ tire, a) raw signal, b) measures scaled.

Les graphiques de la figure 18 représentent l'évolution des forces obtenues sur chaque capteur (moyenne des essais de répétition) en fonction du dosage du mélange.

On remarque sur la figure 18a que les amplitudes des capteurs 1 et 4 croissent avec le pourcentage de sable à l'inverse du capteur 2 , les capteurs 3 et 5 positionnés sur les faces latérales sont sensiblement constants bien que dispersés.

Sur la figure 18b, des moyennes ont été réalisées en fonction de la position des capteurs sur les faces de la cellule. Cette représentation, plus synthétique, permet de constater que pour les mélanges les plus sableux, l'impact est transféré principalement au fond de la cellule. Ce qui est cohérent avec l'augmentation du module du mélange observée lors des essais œdométriques et triaxiaux.

Cette distribution est cohérente avec les observations faites par Haza-Rozier et al. (2010) avec des capteurs d'efforts disposés sur une plaque instrumentée à l'arrière des merlons testés.
La figure 19 présente l'évolution des forces d'impact en fonction du temps pour les différentes modalités. Le montage utilisé pour ces essais ne reproduit que partiellement l'impact d'un bloc sur un élément pareblocs du fait du maintien de la pression dans le vérin après l'impact. Il n'y a pas de décroissance de l'effort après l'atteinte du pic. La chute du signal correspond à la diminution de la pression provoquée par l'action de l'opérateur sur la vanne.

Sinon, on ne voit pas de réelles différences entre les onze expériences. Le temps de réaction des capteurs après l'impact est sensiblement le même. La figure 20 montre que les pentes sont très proches et évoluent de manière identique quels que soient les pourcentages. Les intensités détectées sont les mêmes peu importe le pourcentage de pneu dans l'éprouvette (Fig. 19). 


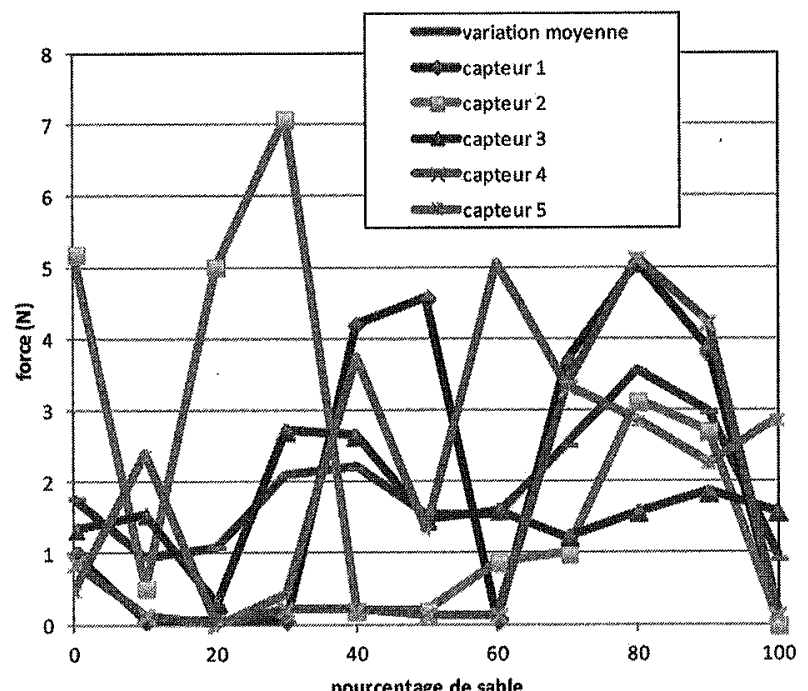

a)

pourcentage de sable

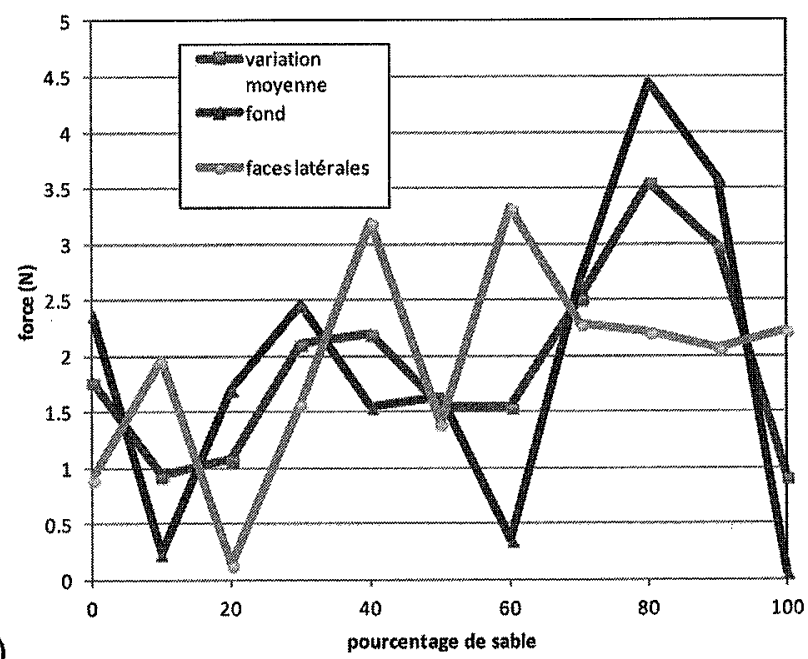

b)

Ail 16 Transfert des efforts dans l'échantillon lors de l'impact.

Transfert of force in the sample during impact.

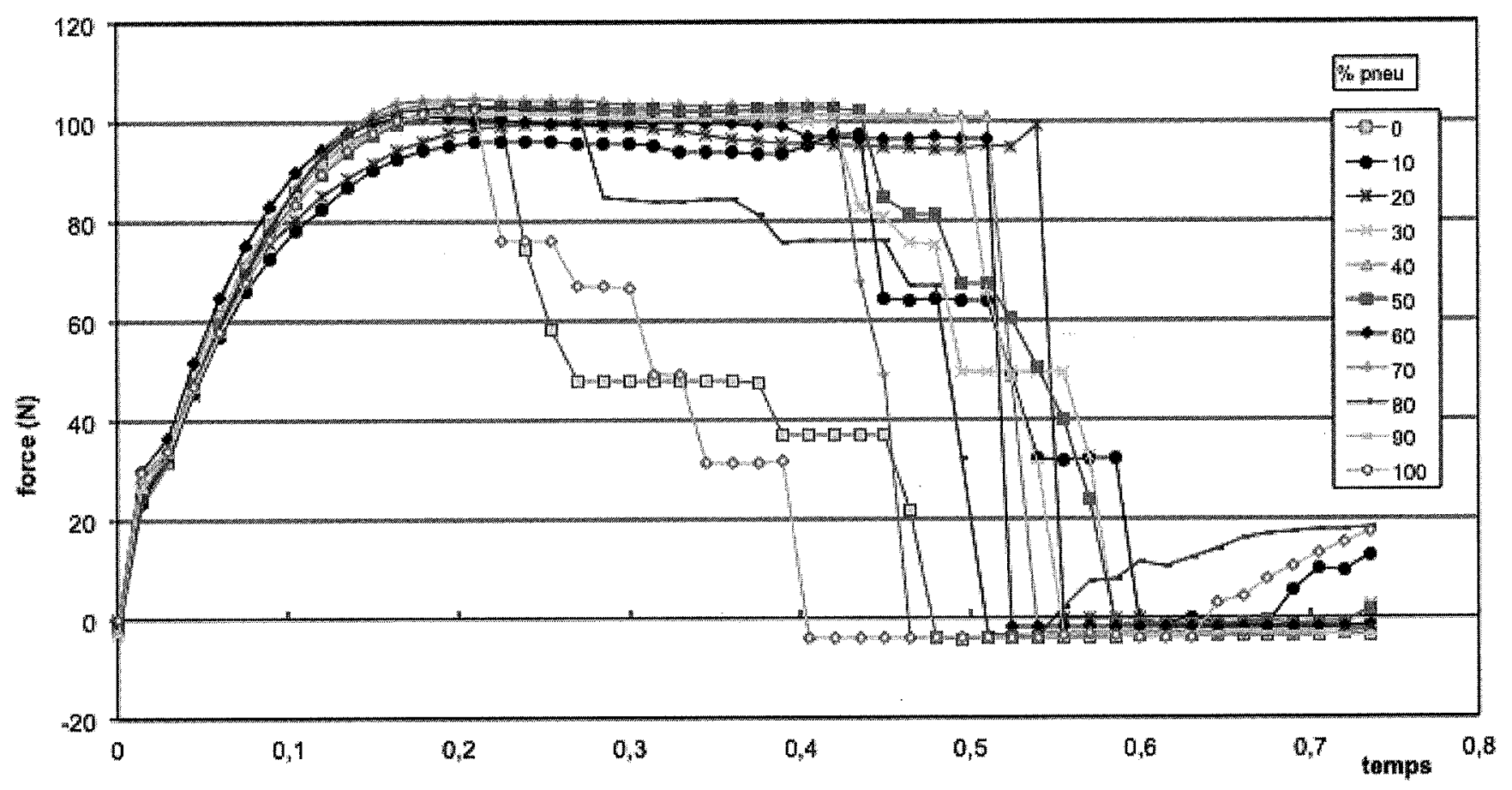

-16 19. Temps de réponse du capteur de force.

Response time of the force sensor.



Pente des courbes d'évolution de la force avec le temps.

Slope of force versus time curves.
5

\section{Conclusion}

L'étude expérimentale exposée dans cet article a permis de constituer une base de données sur le comportement d'éprouvettes reconstituées avec des copeaux de pneumatiques, et du sable dans des conditions d'essais œdométriques, triaxiales et d'impact.

Contrairement à ce qui a été observé sur des mélanges binaires où les deux composantes ont des rigidités proches, les mélanges sable-pneu présentent une capacité d'amortissement, d'un impact, optimum entre 50 et $80 \%$ de matériau le plus grossier (copeaux de pneumatiques) et il est constaté que la résistance au cisaillement est malheureusement plus faible. Il a été 
observé qu'un mélange à environ $60 \%$ de sable (particules fines) et $40 \%$ de copeaux (grosses particules), garantit un arrangement optimal. Il semble donc que plus l'indice des vides au sein de l'éprouvette est petit, meilleurs sont le comportement mécanique et la résistance au cisaillement du mélange. Toutefois, le rapport des dimensions des particules fines et des particules grossières, considérées dans cette étude comme étant respectivement un ballast et des déchiquetats de pneumatiques, donc la forme de la courbe granulométrique régule l'apport d'amortissement et de résistance au cisaillement

Les essais d'impacts réalisés en laboratoire ont validé ces tendances et ont permis de confirmer les observations faites sur les merlons soumis à impacts pendulaires réalisés dans REMPARe. La présence de pneumatique ralentit la montée en efforts à l'intérieur du noyau mais augmente son amplitude.
De plus, il a été constaté lors de nos expériences que la succession d'impacts de même énergie ne densifie pas considérablement les mélanges comportant du pneu, limitant l'effet destructeur de la répétition de l'impact si la structure de confinement résiste.

Il semble donc nécessaire lors du choix du dosage des mélanges amortisseurs destinés à être intégrés dans les merlons pare-blocs de préconiser ces ratios.

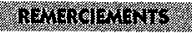

Les auteurs tiennent à remercier le projet ANR REMPARe qui a financé ces expériences et $C$. $-A$. Bonneau, stagiaire au LCPC pour son aide précieuse lors de la réalisation des expérimentations.

\section{Bibliographie}

Bałachowski L., Gotteland P. - Characteristics of Tyre Chips-Sand Mixtures from Triaxial Tests. Archives of Hydro-Engineering and Environmental Mechanics, vol. 54, n 1, 2007, p. 25-36.

Chikatamarla R., Laue J., Springman S. Stress distribution of rockfall events on protection galleries. 16 ICSMGE, Osaka, Balkema Ed., 2005, p. 2789-2792.

Edil T.B., Bosscher P.J. - Engineering Properties of Tyre Chips and Soil Mixtures. Geotechnical Testing Journal, 17 (4), 1994, p. 1-464.

Feng Z.-Y., Sutter K. - Dynamic properties of granulated rubber/sand mixtures. GTJ 23 (3), 2000, p. 338-344.

Gotteland P., Lambert S., Bałachowski L. - Strength characteristics of tyre chipssand mixtures. Studia Geotechnica et Mechanica, vol. XXVII, $\mathrm{n}^{\circ} 1-2,2005$, p. 55-66.

Gotteland P., Lambert S., Salot Ch. - Investigating the Strength characteristics of tyre chips-sand mixtures for geo-cellular structure engineering. Int Workshop on scrap tyre derived geomaterials, Yokosuka, Japan, 2007, 10 p.

Haza-Rozier E., Reiffsteck Ph., Vinceslas G., Lambert S., Haymann A., Gotteland P. Douaillat M. - Tests de merlons amortisseurs sur une station d'essais d'impacts pendulaires. Journées nationales de géotechnique et de géologie de l’ingénieur. JNGG2010, Grenoble 7-9 juillet 2010.

Lambert S. - Comportement mécanique de géocellules; application aux constituants de merlons pare-blocs cellulaires. Thèse de doctorat, Université Joseph-Fourier, Grenoble, 2007, 237 p.

Lambert S., Heymann A., Gotteland P., Douaillat M., Haza-Rozier E., Vinceslas G. - Comparaison expérimentale semi-vraie grandeur du comportement de trois structures pare-blocs. Journées nationales de géotechnique et de géologie de l'ingénieur. JNGG2010, Grenoble 7-9 juillet 2010.

Lade P.V., Liggio C.D., Yamamuro J.A. - Effects of non-plastic fines on minimum and maximum void ratio of sand, GTJODJ, 21 (4), 1998, p. 336-347.

Luong M.P., Eytard J.C., Khay M., Vinceslas G., Papachristou - Transmissibilité vibratoire d'un géomatériau de recyclage. $6^{e}$ colloque national AFPS, École polytechnique, vol. 1, 2003, p. 349-356.

Mavroulidou M., Etan O., Suntharalingam M. - Mechanical properties of granulated tyre rubber-sand mixtures. 11th International conference on environmental science and technology, CEST2009, Chania, Creta, Greece, vol. A 2009, p. 899-906.
Masad E., Taha R., Ho C., Papagiannakis T. - Engineering Properties of Tire/Soil Mixtures as a Lightweight Fill Material. ASTM GTJ, vol. $19, n^{\circ} 3,1996$, p. 1-304.

Mestat Ph. - De la rhéologie des sols à la modélisation des ouvrages géotechniques. Etudes et Recherches des LPC, GT 67, Laboratoires central des ponts et chaussées, 2000, 235 p.

Paikowsky S.G., Hajduk E.L. - Calibration and Use of Grid-Based Tactile Pressure Sensors in Granular Material. Geotechnical Testing Journal, GTJODJ, 20 (2), 1997, p. 218-241.

Reiffsteck Ph., Arbaut J., Sagnard N., Khay M., Subrin D., Chapeau C., Levacher D. - Mesures en laboratoire du comportement mécanique des sols grossiers. Bulletin des Laboratoires des Ponts et Chaussées, $n^{\circ}$ 268-269, 2007, p. 59-82.

Reiffsteck Ph., Nguyen Pham P.T., Arbaut J. - Influence de la répartition granulométrique sur le comportement mécanique d'un sol. Bulletin des laboratoires des ponts et chaussées, $n^{\circ} 268-269,2007$, p. 83-104.

REMPARe - Présentation du projet, 2010, site internet www.rempare.fr.

Zornberg J.G., Cabral A.R., Viratjandr C. - Behaviour of tire shred-and mixtures. Can. Geotech. J., 41, 2004, p. 227-241. 